\title{
ANÁLISE DE ALTERNATIVAS DE TRANSPORTE DE PASSAGEIRO EM RELAÇÃO AO CUSTO E TEMPO DE VIAGEM
}

\section{1- Bianca Cipriano da Silva Zary}

Mestranda em Engenharia de Transportes pelo Instituto Militar de Engenharia (IME), Brasil. Programa de Engenharia de Transportes do Instituto Militar de Engenharia (IME), Brasil. biancabc_rj@yahoo.com.br http://lattes.cnpq.br/9772804282441543

\section{2- Maria Helena Stagi Hossmann}

Mestranda em Engenharia de Transportes pelo Instituto Militar de Engenharia (IME), Brasil. Programa de Engenharia de Transportes do Instituto Militar de Engenharia (IME) mhstagi@yahoo.com.br http://lattes.cnpq.br/5353827373833042

\section{3- Ben-Hur de Albuquerque e Silva}

Doutor em Engenharia Civil pela Universidade Federal do Rio de Janeiro (UFRJ), Brasil. Programa de Engenharia de Transportes do Instituto Militar de Engenharia (IME), Brasil. benhur@ime.eb.br http://lattes.cnpq.br/ 6509325029666357

\section{4- Marcelino Aurélio Vieira da Silva}

Doutor em Engenharia de Transportes pela Universidade Federal do Rio de Janeiro (UFRJ), Brasil. Programa de Engenharia de Transportes do Instituto Militar de Engenharia (IME), Brasil. mauvsil@ime.eb.br

http://lattes.cnpq.br/ 7060585515102803

\author{
Diego Maganhotto Coraiola - Editor Geral \\ Editor responsável pela submissão: \\ Hamilton Pozo. \\ Artigo analisado via processo de revisão duplo cego (Double-blind). \\ Recebido em: 03/02/2014 \\ Aprovado em: 29/04/2014 \\ última Alteração: 18/05/2014
}

* Contato Principal: Praça General Tibúrcio, n 80. Praia Vermelha, Rio de Janeiro - RJ. CEP: 22.290-270. 


\section{ANÁLISE de ALTERNATIVAS DE TRANSPORTE DE PASSAgEIRO EM RELAÇÃo AO CUSTO E TEMPO DE VIAGEM}

\section{Resumo}

O planejamento de transportes deve levar em conta a qualidade de vida da comunidade e de seus usuários. Acredita-se que o custo e a qualidade do serviço ofertado são fatores decisórios no processo de escolha das alternativas pelos usuários. Sendo assim, o estudo apresenta um procedimento para o entendimento da escolha modal de transporte de passageiros com base em dois atributos: tempo e custo de viagem. O objetivo deste trabalho visa, por meio de uma análise de sensibilidade, compreender o provável comportamento de passageiros quanto às suas alternativas, por meio de permutação de opções de transportes coletivo e/ou individual. A aplicação do procedimento metodológico potencializa a compreensão do comportamento de passageiros diante de suas possibilidades disponíveis de transporte. A ferramenta Análise de Sensibilidade permite aos planejadores de transportes perceber que a escolha da alternativa de transporte está intimamente relacionada à renda familiar do indivíduo transportado.

\section{Palavras-Chave}

Transporte urbano individual e coletivo; Alternativas de transporte; Escolha modal; Análise de sensibilidade.

\section{ANALYSIS OF ALTERNATIVE MEANS OF TRANSPORTATION OF PASSENGERS IN RELATION TO THE COST AND TIME TRAVEL}

\section{Abstract}

The transportation planning must take into account the quality of life of the community and its users. It is believed that the cost and the quality of service offered are decisive factors in the selection of alternatives by users. Thus, the study presents a procedure for understanding the transport modal choice of passenger according to two attributes: time and travel cost. This study aims, through a sensitivity analysis, to understand the likely behavior of passengers and their alternatives through permutation of collective and individual options. The application of the methodological procedure enhances the understanding of the behavior of passengers on its available means of transport. The Sensitivity Analysis tool allows transportation planners to realize that the choice of alternative transport is closely related to the individual's family income.

\section{Keywords}

Individual and collective urban transport; Transportation alternatives; Modal choice; Sensitivity analysis. 


\section{Introdução}

A mobilidade precária de algumas metrópoles brasileiras, como o caso de Rio de Janeiro e São Paulo, por suas dimensões geográficas, não tem conseguido de forma satisfatória atender ao direito fundamental das pessoas, que é o de ir e vir. Muito se deve ao crescimento tardio entre as décadas de 1930 e 1980 , fruto da transformação pela qual passou a economia brasileira, de agrária-exportadora para industrializada, gerando movimentos migratórios do tipo campo-cidade (Maciel, 2009).

As classes com menor poder aquisitivo ocuparam locais distantes, conhecidos como periferia, dos locais de emprego, consumo e entretenimento, devido a imensa migração já citada e o encarecimento dos terrenos centrais, melhores localizados. Sendo assim, o preço mais acessível dos terrenos da periferia se tornaram a melhor opção, compensando a baixa acessibilidade e a insuficiência de infraestrutura. E assim, as metrópoles brasileiras foram se distribuindo espacialmente ao longo de décadas (Maciel, 2009).

Com o panorama histórico apresentado, percebe-se que essa situação precária de mobilidade não está apenas vinculada aos atuais gestores, mas sim, ao produto de uma negligência histórica, pois a obrigatoriedade de uma lei de diretrizes para o transporte urbano se fazia presente desde a Constituição Federal de 1988, no entanto a Política Nacional de Mobilidade Urbana só foi sancionada em janeiro de 2012 (Brasil, 2012).

A Política de Mobilidade Sustentável busca atender as necessidades sociais, ambientais e econômicas, sendo assim o planejamento de transportes não deve ser restrito a valores financeiros, pois pode deixar de atender aos outros aspectos referente a sustentabilidade. Pela existência de mais de um aspecto, as ferramentas de análise multicritério podem ajudar na modelagem do problema, seja na hierarquização ou na análise de percentual de escolha para cada alternativa. Uma questão a ser resolvida nos modelos de análise multicritério é a importância de cada aspecto considerado, que na maioria dos métodos é resolvido com consulta a especialista com base em questionário de comparação entre os aspectos.

Devido ao tempo despendido no processo de consulta a especialistas, este artigo tem como hipótese principal conseguir elaborar um procedimento, com base em análise sensibilidade das variáveis envolvidas, para explicar as escolhas dos usuários em alternativas de transporte público.

A premissa do trabalho é que as variáveis tempo de viagem e custo são as variáveis de decisão para os usuários de transportes público para a aplicação realizada. Porém, outras variáveis como conforto, confiabilidade e segurança poderiam ser critérios de escolhas dos usuários, mas não serão testadas neste trabalho.

Um Planejamento de Transportes eficiente é essencial na busca de soluções ou minimizações dos problemas de mobilidade existentes. Diante disso, o presente trabalho busca avaliar o provável processo de escolha modal dos passageiros frente a opções de transporte no percurso da Praia de Icaraí - Niterói para Botafogo - Rio de Janeiro, fazendo uso da Terceira Etapa do Método Sequencial de Quatro Etapas (Bruton, 1979). Propondo um procedimento para análise de alternativas multimodais para o transporte de passageiros no trajeto escolhido para estudo.

Para tanto, será apresentado neste trabalho a avaliação em termos de custo e de tempo de viagem de cada alternativa de intermodalidade. Baseado em uma análise de sensibilidade, o desempenho de cada rota foi avaliado considerando os dois atributos e, assim, tornou possível compreender o comportamento dos passageiros na escolha de alternativa dentro das possíveis opções no trajeto em estudo.

A partir desta introdução, o trabalho está dividido em cinco seções, sendo que a primeira seção que trata do referencial teórico onde a análise de escolha modal e a avaliação de desempenho no transporte são abordadas. Na segunda, terceira e quarta seções estão sendo detalhadas respectivamente a metodologia deste trabalho, a análise de sensibilidade e a análise dos resultados obtidos. A quinta e última parte aborda a conclusão e considerações finais deste artigo.

\section{Revisão Bibliográfica}

\subsection{Análise de Escolha Modal}

Um modelo utilizado e reconhecido pelos planejadores de transportes é o método sequencial de quatro etapas, em que a primeira etapa trata dos modelos de geração de viagens, a segunda etapa de distribuição de viagens, a terceira etapa da divisão ou escolha modal e a quarta etapa da alocação de tráfego (Bruton, 1979; Ortuzar \& Willumsen, 2011).

Inserida na terceira etapa do tradicional Método Sequencial de Demanda, a análise de escolha modal se faz presente quando existir a possibilidade de mais de um modo de transporte levando passageiros e/ou carga de uma determinada origem a um destino final. De uma maneira mais simplista, a divisão modal trata da quantidade de viagens por modo de transporte entre as zonas de tráfego (Campos, 2013).

A escolha modal se apresenta com base em avaliação de atributos característicos de cada modo de transporte. Esses atributos incluem usualmente características sócio-econômicas e características dos serviços dos modos de transporte. Com um enfoque mais generalista, são considerados os atributos do: deslocamento 
(motivo da viagem, período de realização e destino); usuário (propriedade de veículos, renda e estrutura familiar e nível cultural); e sistema de transporte (custo e tempo de viagem, tempo de espera, de transbordo ou andando, frequência, conforto e acessibilidade) (Campos, 2013).

Segundo Bruton (1979), Pereira (2007) e Ortuzar e Willumsen (2011), existem basicamente dois tipos de divisão modal: os determinísticos, que são os métodos quantitativos simples (regressão linear, curvas de desvio e classificação cruzada) e os probabilísticos, que usam mais as formulações matemáticas: LOGIT binomial e multinomial.

A análise da escolha modal, que é um processo diferente da explicação da divisão modal, pode utilizar todas as técnicas de análise multicritério. Segundo Romero (2006), os métodos multicritério podem ser divididos em duas classes, a primeira os Multiobjective Decision Making (tomada de decisão multiobjetivo) que abordam os problemas que têm objetivos múltiplos e fazem parte do ramo contínuo da decisão multicritério. A segunda classe, os Multiattribute Decision Making (tomada de decisão multiatributo) que diz respeito aos métodos em que a solução consiste em encontrar a melhor alternativa baseada em seus atributos e faze, parte do ramo discreto da decisão multicritério (Gomes, Araya \& Carignano, 2004).

Para os problemas de Multiobjective Decision Making (tomada de decisão multiobjetivo) podem ser resolvidos com a aplicação da técnicas de programação por metas, podendo ainda ser divididos nos métodos dos pesos que forma uma única função objetivo que consiste na soma ponderada das metas, e o método hierárquico que otimiza as metas uma por vez começando com a meta de prioridade mais alta e terminando com a de prioridade mais baixa (Taha, 2008).

Para resolver os problemas Multiattribute Decision Making (tomada de decisão multiatributo) existem diversas técnicas como: GRA (Grey Relational Analysis) (Garcia \& Frutuoso e Melo, 2005), DEA (Data Envelopment Analysis) (Silva Neves, 2000), AHP (Analytic Hierarchy Process) (Saaty, 1980), PROMÉTHÉE I, II, III, IV e V (Brans, Mareschal \& Vincke, 1984), ELECTRE | (Roy, 1968), II (Roy \& Bertier, 1973), III (Roy, 1978), IV (Roy \& Hugonnard, 1982), IS (Roy \& Skalka, 1985) e TRI (Yu Wei, 1992), TODIM (Tomada de Decisão Interativa Multicritério) (Fernandes, 1996), MAUT (Multiattribute Utility Theory) (Romero, 2006), TOPSIS (Campos \& Almeida, 2006), VIP Analysis (Variable Interdependent Parameters), MACBETH (Pereira, 2001 ).

O trabalho realizado nesta pesquisa se enquadra nos problemas com multiatributo, porém, para análise não foi utilizada nenhuma das técnicas apresentadas e buscou-se explicar as possíveis escolhas sem utilizar pesquisas com especialistas a partir de análise de sensibilidade das variáveis.

\subsection{Avaliação de Desempenho em Transporte}

A avaliação de desempenho é um ferramental amplamente utilizado pelo mercado privado, com o intuito de se tornar cada vez mais competitivo. Sendo assim, esse instrumento de avaliação pode proporcionar informações que permitam comparar diversas bases de dados, possibilitando levantar tanto os pontos fortes como os fracos do processo em estudo (Oliveira, Borges \& Jabbour, 2005; Gasparetto, Bornia \& Kliemann Neto, 2003).

De acordo com Pratt e Lomax (1996), as medidas de desempenho elaboradas devem focar nos objetivos e metas que determinado sistema de transporte busca atingir, além de levar em consideração, no mesmo grau de importância, a visão do usuário e os impactos para a sociedade de um modo geral.

No entanto, Akasaka, Silva e Leal Jr. (2012) destacam a incipiência de ferramentas adequadas para a avaliação de desempenho em transporte. Com base nessa afirmação, o desenvolvimento de um método com essa característica é válido para aprimorar o processo em questão.

A Tabela 1 apresenta os possíveis indicadores que podem ser utilizados para planejar o sistema de transporte e entender as escolhas dos usuários para cada modo de transportes.

Tabela 1

Indicadores de desenvolvimento sustentável

\begin{tabular}{ccl}
\hline Componente & \multicolumn{1}{c}{ Tema } & \multicolumn{1}{c}{ Indicador } \\
\cline { 2 - 3 } Ambiental & Poluição do ar & $\begin{array}{l}\text { Gases de estufa dos transportes e uso do solo; } \\
\text { Gases acidificados dos transportes e uso do solo; } \\
\text { Composto orgânico dos transportes. }\end{array}$ \\
\cline { 2 - 3 } Consumo de recursos naturais & $\begin{array}{l}\text { Consumo de derivados do petróleo, o consumo de } \\
\text { materiais de construção; } \\
\text { Utilização / ocupação do território pelos transportes e } \\
\text { atividades. }\end{array}$ \\
\cline { 2 - 3 } & Qualidade ambiental & $\begin{array}{l}\text { Indicador de potencial microclimático para a qualidade } \\
\text { da biodiversidade de espaços abertos. }\end{array}$ \\
\hline
\end{tabular}




\begin{tabular}{|c|c|c|}
\hline \multirow{3}{*}{ Social } & Saúde & $\begin{array}{l}\text { Exposição à poluição de partículas geradas pelos } \\
\text { transportes no ambiente vivo; } \\
\text { Exposição ao dióxido de nitrogênio dos transportes no } \\
\text { ambiente vivo; } \\
\text { Exposição ao ruído do tráfego, morte e danos } \\
\text { resultante do tráfego. }\end{array}$ \\
\hline & Equidade & $\begin{array}{l}\text { Justiça na distribuição dos benefícios econômicos; } \\
\text { Justiça na exposição à partículas; } \\
\text { Justiça na exposição ao dióxido de carbono; } \\
\text { Justiça na exposição à emissão de ruídos. }\end{array}$ \\
\hline & Oportunidades & $\begin{array}{l}\text { Tempo total passado no tráfego; } \\
\text { Nível de serviço dos terminais privativos e modos } \\
\text { lentos; } \\
\text { Vitalidade do centro da cidade; } \\
\text { Vitalidade da região circundante; } \\
\text { Acessibilidade ao centro da cidade; } \\
\text { Acessibilidade aos espaços livres; } \\
\text { Feitos sobre o emprego. }\end{array}$ \\
\hline \multirow{3}{*}{ Indicadores econômicos } & $\begin{array}{l}\text { Rede total de benefícios } \\
\text { líquidos do transporte }\end{array}$ & $\begin{array}{l}\text { Benefícios do utilizador de transporte; } \\
\text { Benefícios do operador de transporte; } \\
\text { Custo dos recursos; } \\
\text { Custos externos; } \\
\text { Custos de investimento. }\end{array}$ \\
\hline & $\begin{array}{l}\text { Rede total de benefícios } \\
\text { líquidos do uso do solo }\end{array}$ & $\begin{array}{l}\text { Benefícios do utilizador; } \\
\text { Benefícios do operador; } \\
\text { Custo dos recursos; } \\
\text { Custos externos; } \\
\text { Custos de investimento. }\end{array}$ \\
\hline & $\begin{array}{c}\text { Economia regional e } \\
\text { competitividade }\end{array}$ & (Não especificado) \\
\hline
\end{tabular}

Fonte: Campos (2013).

Para o desenvolvimento deste trabalho, foi utilizado o custo de cada modo e o valor do tempo como sendo os atributos a serem utilizados na análise.

\section{Método de Pesquisa e Procedimento Técnico para Análise de Escolha Modal de Transporte de Passageiros}

Para este estudo, serão apresentados a seguir, segundo a metodologia de Silva e Menezes (2001), a classificação e o procedimento metodológico utilizado.

\subsection{Classificação da Pesquisa}

Segundo Silva E Menezes (2001), A Pesquisa Pode Ser Classificada De Forma Clássica Quanto: A Sua Natureza, Ao Ponto De Vista Da Forma De Abordagem Do Problema, O Ponto De Vista Dos Seus Objetivos E Do Ponto De Vista Do Procedimento Técnico. Desta Forma, O Estudo Desenvolvido Se Enquadra Em Uma Pesquisa De Natureza Aplicada Devido A Busca Na Geração De Conhecimentos Para Aplicação Prática Dirigida À Solução De Problemas Específicos No Transporte De Passageiros.

Quanto À Segunda Classificação, A Pesquisa É Definida Como Um Estudo Quantitativo, Na Abordagem Do Problema, Uma Vez Que Considera-Se Que Os Dados Podem Ser Quantificáveis Com A Utilização Técnica Estatística (Análise De Sensibilidade).

Quanto À Terceira Classificação, À Luz Dos Seus Objetivos, O Estudo É Classificado Como Uma Pesquisa Exploratória, Pois Há Um Aprofundamento Do Problema De Pesquisa Tratado E Um Detalhamento Dos Passos Para A Solução Do Mesmo.

De Acordo Com Gil (1991), Quanto Aos Procedimentos De Solução, O Estudo Pode Ser Dividido Em Pesquisa Bibliográfica E Experimental. Primeiramente, Buscou-Se O Embasamento Dos Conceitos Necessários Para Estruturação E Desenvolvimento Da Pesquisa.

Por Fim, Uma Pesquisa Experimental Foi Realizada No Trecho Praia De Icaraí - Botafogo, Uma Vez Que, Determinou-Se Um Objeto De Estudo, Selecionaram-Se As Variáveis Que Seriam Capazes De Influenciá-Lo, Definiram-Se As Formas De Controle E De Observação Dos Efeitos Que A Variável Produz No Objeto. 


\subsection{Procedimento Metodológico}

A Figura 1 apresenta um fluxograma com o procedimento proposto para avaliar as alternativas modais, considerando as categorias tempo e custo de transporte, para o usuário.

\begin{tabular}{|c|c|c|c|}
\hline ETAPA 1 & ETAPA 2 & ETAPA 3 & ETAPA 4 \\
\hline $\begin{array}{c}\text { Definição da origem } \\
\text { e destino e } \\
\text { alternativas. }\end{array}$ & $\begin{array}{l}\text { Levantamento e } \\
\text { definição do custo. }\end{array}$ & $\begin{array}{l}\text { Levantamento e } \\
\text { definição do tempo } \\
\text { de viagem dos }\end{array}$ & $\begin{array}{c}\text { Análise de } \\
\text { sensibilidade. }\end{array}$ \\
\hline $\begin{array}{l}\text { SAÍDA } \\
\text { Pares definidos. }\end{array}$ & $\begin{array}{l}\text { SAÍDA } \\
\text { Custo definido. }\end{array}$ & $\begin{array}{l}\text { SAÍDA } \\
\text { Tempo definido. }\end{array}$ & $\begin{array}{l}\text { SAÍDA } \\
\text { Sensibilidade } \\
\text { realizada. }\end{array}$ \\
\hline
\end{tabular}

Figura 1 - Procedimento proposto Fonte: Autoria própria.

Respeitando o fluxograma proposto na Figura 1, foi possível passar para o estudo de caso. E para um melhor entendimento, o estudo em questão foi dividido nos quatro subitens (passos) a seguir:

\subsubsection{Definição de origem e destino e alternativas (etapa 1)}

A busca por uma origem e um destino que permitissem gerar diversos percursos diferentes e com opções de três modos (rodoviário, metroviário e hidroviário), levou à escolha da Praia de Icaraí, em Niterói, como origem e Botafogo, no Rio de Janeiro, como destino. A baía de Guanabara separa estas duas cidades, possibilitando ampliar o número de opções de transportes pelo usuário através do transporte hidroviário. Já, no Rio de Janeiro, o percurso entre o Centro e Botafogo possui as opções do metrô e do ônibus.

Definidos origem e destino, foram levantadas as possíveis combinações de modos, o que acarretou em oito alternativas que englobam transportes coletivos e/ou transportes individuais, conforme a Tabela 2.

Tabela 2

Alternativas de transporte para O/D Icaraí $\rightarrow$ Botafogo

\begin{tabular}{cccccc}
\hline Alternativa & Modo 1 & & Modo 2 & & Modo 3 \\
\hline A 1 & Ônibus & & & & \\
\hline A 2 & Carro & & & & \\
\hline A 3 & Táxi & & & & \\
\hline A 4 & Ônibus & $\rightarrow$ & Barca & $\rightarrow$ & Ônibus \\
\hline A 5 & Ônibus & $\rightarrow$ & Barca & $\rightarrow$ & Metrô \\
\hline A 6 & Ônibus & $\rightarrow$ & Barca & $\rightarrow$ & Táxi \\
\hline A 7 & Táxi & $\rightarrow$ & Barca & $\rightarrow$ & Táxi \\
\hline A 8 & Táxi & $\rightarrow$ & Barca & $\rightarrow$ & Ônibus \\
\hline
\end{tabular}

Fonte: Autoria própria.

\subsubsection{Levantamento e definição do custo (etapa 2)}

Para compor o custo total de cada alternativa faz-se necessário levantar o custo de cada modo de transporte separadamente. Para isto, primeiramente, foram levantados os dados do transporte coletivo. Com base no PDTU (2013), foi possível levantar esses custos unitários. Já para o transporte intermunicipal (alternativa A1), o custo da tarifa foi obtido por meio de contato telefônico com a empresa Auto Viação 1001, solicitando o valor cobrado por passageiro quando utilizando a linha $775 \mathrm{~d}$.

Considerando o transporte individual, o processo para obtenção da tarifa se deu da seguinte forma:

Alternativa Carro $\rightarrow \mathrm{O}$ valor considerado para os custos operacionais de automóveis é de $\mathrm{R} \$ 0,78$ por $\mathrm{km}$ percorrido (PDTU, 2013). E utilizando o software TRANSCAD, foi possível calcular a distância e, por conseguinte, o valor gasto pela alternativa em estudo.

Alternativa Táxi $\rightarrow$ Para o valor da tarifa nos modais onde se fez presente a opção do táxi, foram utilizados sites de cálculo de tarifa de táxi para os municípios de Niterói e Rio de Janeiro (COOPTAX, 2013 e TARIFA DE TÁXI, 2013).

Com os dados levantados, foi possível montar a Tabela 3, que apresenta o valor encontrado de cada modo e o custo total de cada alternativa. 
Tabela 3

Custo de tarifa (R\$) para O/D Icaraí $\rightarrow$ Botafogo

Fonte: Autoria própria.

\begin{tabular}{ccccc}
\hline Alternativa & Modo 1 & Modo 2 & Modo 3 & Custo Total \\
\hline A1 & $\mathrm{R} \$ 5,95$ & & & $\mathrm{R} \$ 5,95$ \\
\hline $\mathrm{A} 2$ & $\mathrm{R} \$ 19,32$ & & & $\mathrm{R} \$ 19,32$ \\
\hline $\mathrm{A} 3$ & $\mathrm{R} \$ 56,50$ & & & $\mathrm{R} \$ 56,50$ \\
\hline $\mathrm{A} 4$ & $\mathrm{R} \$ 2,75$ & $\mathrm{R} \$ 4,80$ & $\mathrm{R} \$ 2,75$ & $\mathrm{R} \$ 10,30$ \\
\hline $\mathrm{A} 5$ & $\mathrm{R} \$ 2,75$ & $\mathrm{R} \$ 4,80$ & $\mathrm{R} \$ 3,50$ & $\mathrm{R} \$ 11,05$ \\
\hline $\mathrm{A} 6$ & $\mathrm{R} \$ 2,75$ & $\mathrm{R} \$ 4,80$ & $\mathrm{R} \$ 17,96$ & $\mathrm{R} \$ 25,51$ \\
\hline $\mathrm{A} 7$ & $\mathrm{R} \$ 9,70$ & $\mathrm{R} \$ 4,80$ & $\mathrm{R} \$ 17,96$ & $\mathrm{R} \$ 32,46$ \\
\hline $\mathrm{A} 8$ & $\mathrm{R} \$ 9,70$ & $\mathrm{R} \$ 4,80$ & $\mathrm{R} \$ 2,75$ & $\mathrm{R} \$ 17,25$ \\
\hline
\end{tabular}

\subsubsection{Levantamento e definição do tempo de viagem dos modos (etapa 3)}

Da mesma forma que no subitem 3.2.2, faz-se necessário levantar o tempo gasto de cada modo de transporte separadamente. Porém no caso de tempo gasto em transporte, é necessário considerar além do tempo efetivo em cada modo, os tempo referentes ao período de espera, ao tempo de transbordo e ao tempo de caminhada entre modos de transporte ou entre o modo de transporte e o destino final.

O levantamento do tempo gasto por cada alternativa foi feito, primeiramente, obtendo o percurso de cada meio de transporte, através de buscas na internet.

As empresas pesquisadas foram:

- Auto Viação 1001 (2013), linha 775d, para a alternativa 1;

- Auto Lotação Ingá (2013), linha 49, para o modo 1 das alternativas 4, 5 e 6;

- Braso Lisboa (2013), linha 474, para o modo 3 das alternativas 4 e 8;

- CCR Barcas (2013) para o modo 2 das alternativas 4, 5, 6, 7 e 8;

- Metrô Rio (2013) para o modo 3 da alternativa 5;

- COOPTAX (2013), para o modo 1 das alternativas 3, 7 e 8;

- TARIFA DE TÁXI (2013), para o modo 3 das alternativas 6 e 7.

Alternativa Ônibus $\rightarrow$ Para o transporte intermunicipal (alternativa 1 ) e para os transportes municipais (alternativas 4, 5, 6 e 8) foi feito o levantamento do tempo gasto entre a origem e o destino através do Google mapas (2013) opção carro, seguindo o itinerário de cada linha e acrescendo $20 \%$ ao valor total em função das paradas. O tempo de transbordo das linhas foi definido com a pesquisa dos intervalos entre os ônibus, através dos sites de suas empresas, e tirada a média desses valores. Para a linha intermunicipal foi definido um intervalo entre carros de 40 minutos, gerando uma média de 20 de espera. Tanto as linhas municipais de Niterói quanto do Rio de Janeiro apresentaram um tempo intervalo entre carros de 3 minutos, gerando um tempo médio de 1,5 minutos para cada linha.

Alternativa Táxi $\rightarrow$ Para o levantamento do tempo gasto pelos táxis (alternativas 3, 6, 7 e 8) pesquisouse nos sites das empresas de táxi de Niterói e do Rio de Janeiro. Definindo-se a origem e o destino desejado obtém-se o tempo total e a distância total percorrida pelo modal (COOPTAX, 2013 e TARIFA DE TÁXI, 2013).

Alternativa Barcas $\rightarrow$ O levantamento do tempo gasto na travessia da Baia de Guanabara pelas barcas (alternativas $4,5,6,7$ e 8) foi feito através de pesquisa no site da empresa CCR Barcas (2013), onde são disponibilizados todos os horários de partidas e chegadas. O tempo de transbordo foi retirado do PDTU (2013), onde é apresentado o tempo de espera entre uma embarcação e outra de 10 minutos, mais o tempo para embarcar de 5 minutos, e tirada a média entre eles.

Alternativa Metrô $\rightarrow$ O levantamento do tempo gasto entre as estações da Carioca e de Botafogo foi definido com a ajuda do Google Mapas (2013). Após inserir a origem (estação da Carioca) e o destino (estação de Botafogo) e optando-se pelo transporte público, 
é gerado o percurso da linha do metrô com a distância e o tempo total, que no caso em questão foi de 23 minutos. O tempo de transbordo foi retirado do PDTU (2013), onde se pode coletar o tempo de intervalo entre as composições de 6 minutos, gerando um tempo médio de 3 minutos.

Alternativa Carro $\rightarrow$ O levantamento do tempo gasto pelo carro (alternativa 2) foi feito através do Google Mapas (2013); preenchendo-se a origem e o destino com a opção carro obtémse o tempo e a distância totais.

Caminhada $\rightarrow$ Para gerar o tempo de caminhada entre um transporte e outro foi utilizado o Google Mapas (2013), preenchendo-se a origem e o destino desejados com a opção a pé. Com isso, encontramos para as alternativas 4, 5 e 6, um tempo de caminha de 4,12 minutos entre o modal 1 e o modal 2. Para as alternativas $4,6,7$ e 8 foi encontrado o tempo de caminhada de 2 minutos entre a saída da estação das barcas da Praça XV e a entrada no "mergulhão", onde existem pontos de ônibus e táxis. Para a alternativa 5 foi encontrado o tempo de caminhada de 10 minutos entre a estação das barcas da Praça XV e a estação de metrô da Carioca, na Avenida Rio Branco.

As Tabelas 4 e 5 mostram, respectivamente, o tempo gasto por cada modo e o tempo de transferência e calculado ao final o tempo total de cada alternativa.

Tabela 4

Tempo total nos modos de transporte (min) para O/D Icaraí $\rightarrow$ Botafogo

Fonte: Autoria própria.

\begin{tabular}{ccccc}
\hline Alternativa & Modo 1 & Modo 2 & Modo 3 & Tempo Total \\
\hline A1 & 68,40 & & & 68,40 \\
\hline A2 & 29,00 & & & 29,00 \\
\hline A3 & 29,00 & & & 29,00 \\
\hline A4 & 6,00 & 20,00 & 35,00 & 61,00 \\
\hline A5 & 6,00 & 20,00 & 23,00 & 49,00 \\
\hline A6 & 6,00 & 20,00 & 9,00 & 35,00 \\
\hline A7 & 4,00 & 20,00 & 9,00 & 33,00 \\
\hline A8 & 4,00 & 20,00 & 35,00 & 59,00 \\
\hline
\end{tabular}

Tabela 5

Tempo total de cada alternativa (min) para O/D Icaraí $\rightarrow$ Botafogo

\begin{tabular}{|c|c|c|c|c|c|c|c|c|}
\hline \multirow{2}{*}{ Alternativa } & \multicolumn{2}{|c|}{$\Delta \mathrm{t}_{0 \rightarrow 1}$} & \multicolumn{2}{|c|}{$\Delta \mathrm{t}_{1 \rightarrow 2}$} & \multicolumn{2}{|c|}{$\Delta \mathrm{t}_{2 \rightarrow 3}$} & \multirow{2}{*}{ Tempo Total de Transf } & \multirow{2}{*}{ Transf + Modal } \\
\hline & Espera & Caminhada & Transb & Caminhada & Transb & Caminhada & & \\
\hline $\mathrm{A} 1$ & 20,00 & - & - & - & - & - & 20,00 & 88,40 \\
\hline $\mathrm{A} 2$ & - & - & - & - & - & - & 0,00 & 29,00 \\
\hline $\mathrm{A} 3$ & - & - & - & - & - & - & 0,00 & 29,00 \\
\hline A4 & 1,50 & 4,12 & 10,00 & 2,00 & 1,50 & - & 19,12 & 80,12 \\
\hline A5 & 1,50 & 4,12 & 10,00 & 10,00 & 3,00 & - & 28,62 & 77,62 \\
\hline A6 & 1,50 & 4,12 & 10,00 & 2,00 & - & - & 17,62 & 52,62 \\
\hline A7 & - & - & 10,00 & 2,00 & - & - & 12,00 & 45,00 \\
\hline A8 & - & - & 10,00 & 2,00 & 1,50 & - & 13,50 & 72,50 \\
\hline
\end{tabular}

Fonte: Autoria própria. 


\subsubsection{Análise de sensibilidade (etapa 4)}

Com a definição do tempo total e do custo total por alternativa, torna-se primordial para a análise de sensibilidade normalizar os resultados obtidos, tanto para o custo da tarifa quanto para o tempo de viagem, uma vez que os valores possuem muita disparidade e podem tornar-se inconsistentes.

Sendo assim, para a normalização dos dados utilizou a equação 1 , buscando resultados de custo e tempo no intervalo próximo a zero e um. Para isso, com $n=2$ (para a tarifa) e $n=1$ (para o tempo), foi possível obter o custo normalizado e o tempo normalizado entre as oito opções, possibilitando a análise do comportamento entre elas, conforme pode ser visto na Tabela 6.

$N=\frac{V}{M+n \times \delta}$

em que:

N é o valor normalizado;

V é o valor a ser normalizado;

M é a média aritmética do intervalo a ser normalizado;

n é uma constante que multiplica o desvio padrão que deverá ser determinada na aplicação do procedimento; e $\delta$ é o desvio padrão.

Tabela 6

Normalização de tarifa e custo para O/D Icaraí $\rightarrow$ Botafogo

\begin{tabular}{|c|c|c|c|c|}
\hline \multirow{2}{*}{ Alternativa } & \multirow{2}{*}{ Tarifa total } & \multirow{2}{*}{ Tempo Total } & \multicolumn{2}{|c|}{ Custo e tempo normalizados } \\
\hline & & & $\mathrm{C} \%$ & $\mathrm{~T} \%$ \\
\hline $\mathrm{A} 1$ & $\mathrm{R} \$ \quad 5,95$ & 88,40 & 0,113 & 1,087 \\
\hline $\mathrm{A} 2$ & $\mathrm{R} \$ 19,32$ & 29,00 & 0,366 & 0,357 \\
\hline $\mathrm{A} 3$ & $\mathrm{R} \$ 56,50$ & 29,00 & 1,071 & 0,357 \\
\hline A4 & $\mathrm{R} \$ 10,30$ & 80,00 & 0,195 & 0,986 \\
\hline A5 & $\mathrm{R} \$ 11,05$ & 78,00 & 0,209 & 0,955 \\
\hline A6 & $\mathrm{R} \$ 25,51$ & 53,00 & 0,484 & 0,647 \\
\hline A7 & $\mathrm{R} \$ 32,46$ & 45,00 & 0,615 & 0,554 \\
\hline A8 & $\mathrm{R} \$ 17,25$ & 73,00 & 0,327 & 0,892 \\
\hline Média & $\mathrm{R} \$ 22,29$ & 59,28 & \multirow{2}{*}{$n=2$} & \multirow{2}{*}{$n=1$} \\
\hline Desvio Padrão & $\mathrm{R} \$ 15,23$ & 22,01 & & \\
\hline
\end{tabular}

Fonte: Autoria própria.

Definida a normalização, é possível avançar para a análise de sensibilidade propriamente dita. Conforme premissa do estudo, a função utilidade, responsável por determinar o grau de satisfação que o usuário do transporte obtém com a escolha do modo, é função das variáveis atributo custo e tempo de viagem. Sendo assim, essa análise de sensibilidade se processou variando os pesos do custo da tarifa, como também o do tempo. De uma forma mais simples, essa ponderação ganhou forma na Tabela 7, onde cada célula foi preenchida de acordo com a função utilidade apresentada pela equação 2, adaptada de Novaes (1986), onde os atributos considerados foram tempo e custo de viagem.

$U_{i}=C_{N} \times C \%+T_{N} \times T \%$

em que:

$U_{i}$ é a função utilidade na alternativa i $\left(A_{i}\right)$, onde i varia de 1 a 8 ;

$\mathrm{C}_{\mathrm{N}}$ é o custo normalizado;

C\% é o peso da variável custo; 
$\mathrm{T}_{\mathrm{N}}$ é o tempo normalizado; e

T\% é o peso da variável tempo.

Na Tabela 7 estão destacadas, em negrito e itálico, as duas melhores opções, respectivamente, escolhidas pela análise de sensibilidade para cada percentual variado.

Tabela 7

Variação de peso do custo e tempo para O/D Icaraí $\rightarrow$ Botafogo

\begin{tabular}{cccccccc}
\hline \multicolumn{7}{c}{ Variação de peso do custo e tempo } \\
\hline $\mathrm{C} \%$ & $100 \%$ & $90 \%$ & $70 \%$ & $50 \%$ & $30 \%$ & $10 \%$ & $0 \%$ \\
\hline $\mathrm{T} \%$ & $0 \%$ & $10 \%$ & $30 \%$ & $50 \%$ & $70 \%$ & $90 \%$ & $100 \%$ \\
\hline $\mathrm{A} 1$ & 0,113 & 0,210 & $\underline{0,405}$ & 0,600 & 0,795 & 0,990 & 1,087 \\
\hline $\mathrm{A} 2$ & 0,366 & 0,365 & 0,363 & 0,361 & 0,359 & 0,358 & 0,357 \\
\hline A3 & 1,071 & 0,999 & 0,857 & 0,714 & $\underline{0,571}$ & $\underline{0,428}$ & $\underline{0,357}$ \\
\hline A4 & $\underline{0,195}$ & $\underline{0,274}$ & 0,432 & 0,5902 & 0,748 & 0,906 & 0,985 \\
\hline A5 & 0,209 & 0,284 & 0,433 & 0,582 & 0,731 & 0,880 & 0,955 \\
\hline A6 & 0,484 & 0,499 & 0,533 & $\underline{0,565}$ & 0,598 & 0,631 & 0,647 \\
\hline A7 & 0,615 & 0,609 & 0,597 & 0,584 & 0,572 & 0,560 & 0,554 \\
\hline A8 & 0,327 & 0,383 & 0,496 & 0,609 & 0,722 & 0,835 & 0,892 \\
\hline
\end{tabular}

Fonte: Autoria própria.

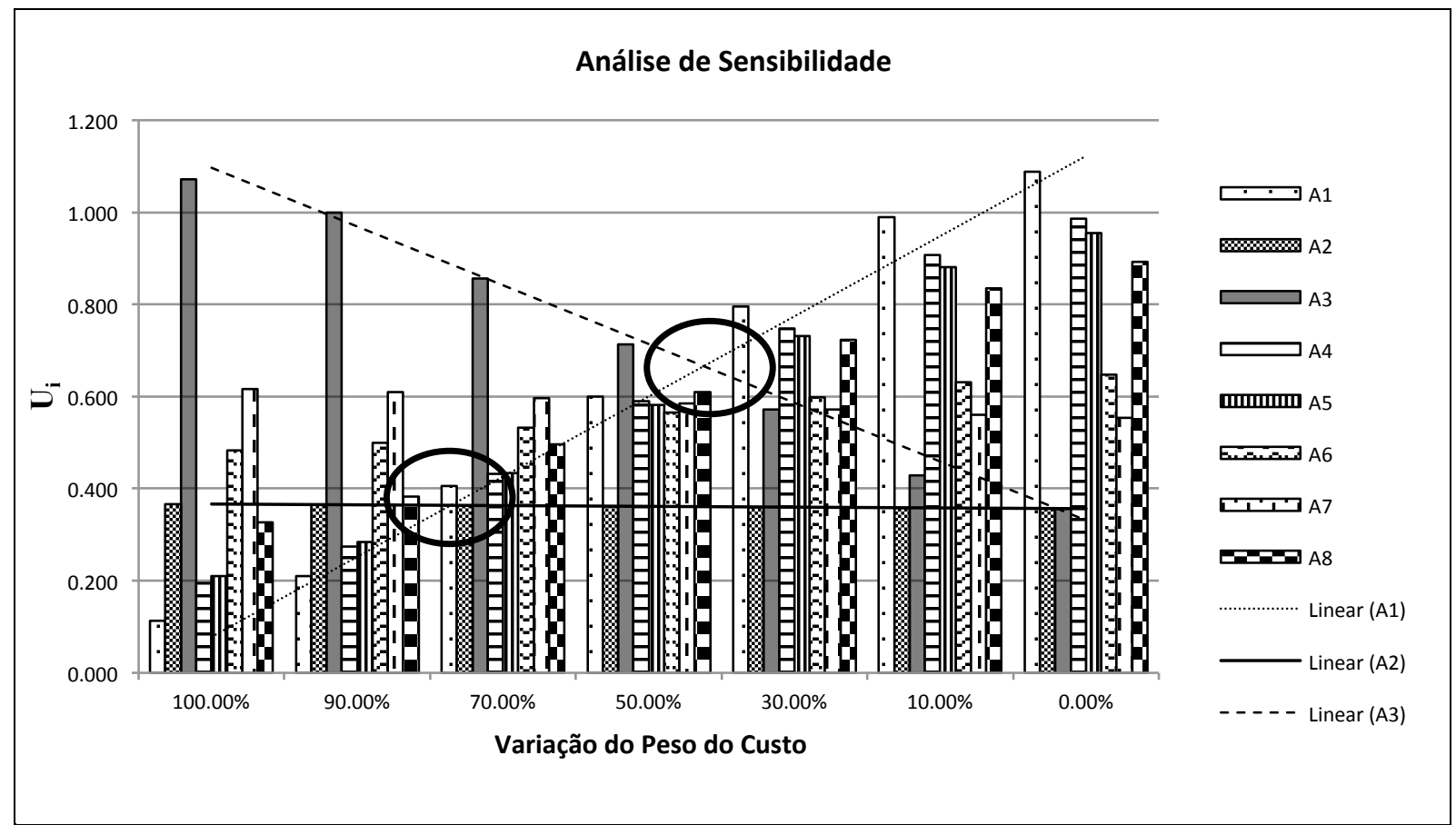

Figura 2. Análise de Sensibilidade

Fonte: Autoria própria.

\section{Análise de Resultados}

Por meio da análise de sensibilidade (Figura 2) realizada no item a cima, se faz possível analisar os resultados e percebe-se um acréscimo acentuado na alternativa Al e um decréscimo acentuado na alternativa A3. Isso se deve à diferença significativa de tempo gasto no percurso e no custo entre esses dois modos. Ainda analisando a Figura 2, os pontos em destaque marcados, interseção da linha de tendência (A1) com as linhas de tendência (A2 e A3) traçadas, equivalem aos pontos onde há uma mudança comportamental. Isto é, a alteração do transporte coletivo pelo individual, uma vez que as linhas de tendência fazem referência as alternativas A1 e A2/A3.

Avaliando-se ainda a Figura 2 em conjunto com a Tabela 7, fica claro que as alternativas que possuem transporte individual (A2, A3, A6, A7 e A8), ou em sua totalidade ou parcialmente, foram as que mais se 
destacaram quando maior peso se deu ao custo. Logo tornou o transporte unicamente coletivo (A1, A4, A5), como a opção mais lucrativa (menores custos).

Entretanto, quando a mesma análise é feita, porém agora dando maior peso ao tempo, observa-se a mudança comportamental, onde o transporte coletivo (A1, A4, A5, A6, A7 e A8), ou em sua totalidade ou parcialmente, foi o que mais se destacou. Logo tornou o transporte unicamente individual (A2 e $A 3$ ), como a opção mais rápida (menores tempos).

Com essa análise, foi possível perceber que a primeira mudança comportamental, da migração do transporte coletivo para o individual, se deu quando a importância ao custo foi entre a porcentagem de 70 e 90 , conforme Tabela 7.

Pode-se também, além dessas análises, observar que dentre as alternativas de transporte unicamente coletivo (A1, A4, A5) e unicamente individual (A2 e A3), apenas a alternativa (A2), onde foi utilizado o carro como modo de transporte, apresentou uma linha de tendência praticamente paralela ao eixo horizontal (Figura 2). Com o coeficiente angular da reta próximo a zero, pode-se constatar que a alternativa $A 2$ foi pouco sensível a variação dos atributos custo e tempo, devendo a alternativa em questão ter outros atributos qualitativos que diferenciasse essa escolha das demais, como por exemplo: conforto, confiabilidade, segurança e privacidade.

Com base no PDTU (2013), os deslocamentos devido ao trabalho ocupam o segundo patamar em importância, só perdendo posição para as viagens por motivo de residência. A Tabela 8 apresenta os dados que serviram de base para uma análise quanto à renda e ao modo de transporte utilizado, onde SM corresponde ao salário mínimo. Os indivíduos que decidem pela escolha dessas alternativas possuem valores para os atributos custo e tempo diferenciado que pode ser explicado pela sua renda. A variação da escolha modal pela renda pode ser visto na Figura 3.

Tabela 8

Modo de transporte por classe de renda (SM - salário mínimo) segundo o motivo trabalho

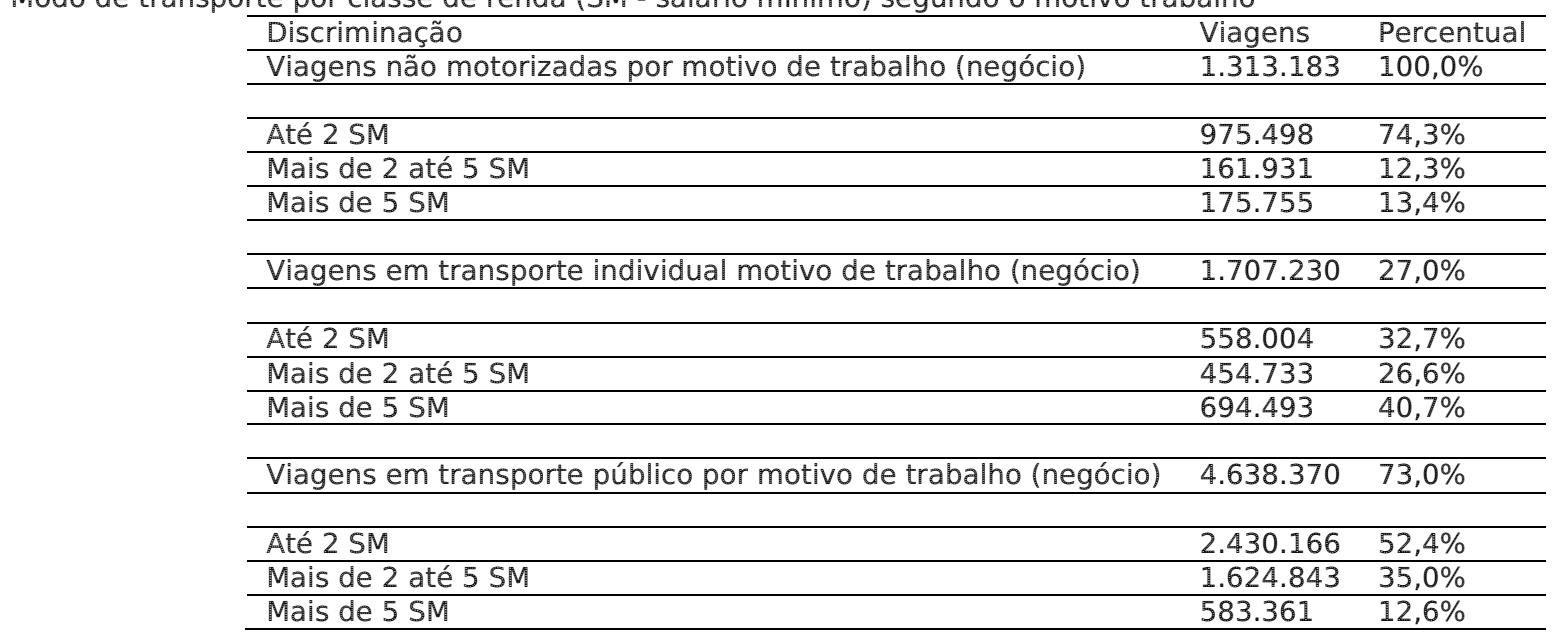

Fonte: PDTU (20)

\section{Renda Familiar per capita mensal}

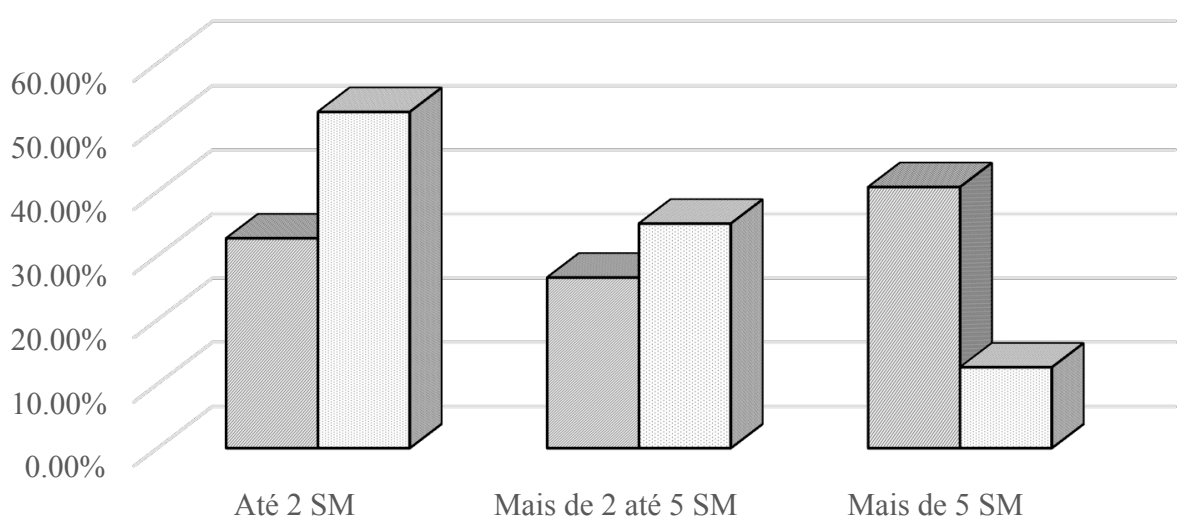

ĐTransporte individual $\square$ Transporte público

Figura 3. Modos motorizados por classe de renda para viagens motivo trabalho

Fonte: PDTU (2013). 


\section{Conclusões}

A escolha dos modos de transporte nos grandes centros urbanos influi diretamente no cotidiano das pessoas. Seja no transporte de passageiros, seja no de carga, a decisão de se realizar o deslocamento está associada a fatores como: o custo de deslocamento, o tempo de viagem, o nível de conforto e de qualidade do transporte, além da oportunidade e possibilidade da sua realização. E são esses fatores associados de forma ponderada que permitem ao usuário a definição: de realizar ou não o deslocamento, da escolha do modo ou modos a utilizar e do momento para a realização do deslocamento.

Com isso exposto, o trabalho em questão teve como finalidade analisar o provável comportamento de passageiros quanto a sua escolha modal, por meio de permutação de opções de transportes coletivo e/ou individual. Sendo assim, a análise de sensibilidade realizada no trabalho apresentou comportamentos mais detalhados das variáveis (tempo e custo).

Por meio do PDTU (2013), evidenciou-se que a classe de renda influencia diretamente no peso das variáveis estudadas. A informação gerada se torna pertinente para auxiliar a tomada de decisão em ambientes de escolha de alternativas de transportes.

No estudo apresentado, a alternativa A2 (carro foi o meio de locomoção) se apresentou pouco sensível as variáveis analisadas. Sendo assim, como proposta deste estudo para trabalhos futuros, recomenda-se que sejam buscadas outras variáveis que expliquem a escolha modal como por exemplo: conforto, confiabilidade, segurança e privacidade.

A utilização de outros atributos e o levantamento de seus respectivos indicadores, mesmos os qualitativos e os ambientais, podem ajudar a explicar e planejar a divisão modal em uma região.

\section{Notas}

1. Os autores deixam expressos seus sinceros agradecimentos aos avaliadores, que por meio de suas recomendações, contribuíram de forma efetiva para o aprimoramento do trabalho.

\section{Referências}

Akasaka, Y.; da Silva, M. A. V. \& Leal Jr., I. C. (2012) Procedimento de escolha e análise de alternativas de transporte para exportação de bioetanol no Brasil. XXVI ANPET - Congresso de Ensino e Pesquisa em Transporte, 2012 - Joinville, Santa Catarina, Brasil

Auto Lotação Ingá (2013). Disponível em:< http://www.setrerj.org.br/linhas/mun_nit_49.html >. Acesso em: 12 de dezembro de 2013.

Auto Viação 1001 (2013). Disponível em:< http://www.autoviacao1001.com.br/pt/ >. Acesso em: 12 de dezembro de 2013.

BRASIL (2012). Lei no 12.587, de 03 de janeiro de 2012. Institui as diretrizes da Política Nacional de Mobilidade Urbana. Diário Oficial da República Federativa do Brasil, Brasília, DF.

Brans, J. P., Mareschal, B. \& Vincke, Ph., 1984, PROMOTHEE: a new family of outranking methods in multicriteria analysis, Operational Research '84, Amsterdam: Elsévier, pp. 408-421.

Braso Lisboa (2013). Disponível em:< http://www.brasolisboa.com.br/itinerarios_474.htm >. Acesso em: 12 de dezembro de 2013.

Bruton, M. J. (1979). Introdução ao planejamento dos transportes. São Paulo: Editora da Universidade de São Paulo.

Campos, V. (2013). Planejamento de Transportes - Conceitos e Modelos. Rio de Janeiro: Interciência.

Campos, V. R. \& ALMEIDA, A. T. (2006). Modelo multicritério de decisão para localização de Nova Jaguaribara com VIP Analysis. Pesquisa Operacional, v.26, n.1, p.91-107, Janeiro a Abril de 2006.

CCR Barcas (2013). Disponível em:< http://www.grupoccr.com.br/barcas/ >. Acesso em: 12 de dezembro de 2013.

COOPTAX (2013). Disponível em:< http://www.cooptax.com.br/calculo.html>. Acesso em: 12 de dezembro de 2013.

Fernandes, C. H. (1996) Priorização de projetos hidrelétricos sob a ótica social: um estudo de caso utilizando análise custo/benefício e uma metodologia multicritério de apoio à decisão - "MACBETH". Dissertação de mestrado - Programa de Pós-Graduação em Engenharia de Produção, Universidade Federal de Santa Catarina, Florianópolis.

Garcia, P. A. A. \& Frutuoso e Melo, P. F. F. (2005), Aplicação de um modelo Grey DEA à análise comparativa de riscos entre tecnologias de geração de energia. International Nuclear Atlantic Conference - INAC 2005, Santos, 
SP, Brasil.

Gasparetto, V., Bornia, A. C. e Kliemann Neto, F. J., 2003, Análise Crítica de Sistemáticas de Avaliação de Desempenho de Cadeias de Suprimentos. Anais do VIII Congresso del Instituto Internacional de Costos, Punta del Este, Uruguai: IIC.Gil, A. C. (1991). Como elaborar projetos de pesquisa. São Paulo: Atlas.

Gomes, L. F. A. M., Araya, M. C. G. \& Carignano, C. (2004) Tomada de decisões em cenários complexos: introdução aos métodos discretos do apoio multicritério à decisão - São Paulo: Pioneira Thomson Learning.

Google Mapas (2013). Disponível em:< https://maps.google.com.br/ >. Acesso em: 16 de dezembro de 2013.

Maciel, V. F. (2009) Problemas e desafios do transporte público urbano. Disponível em:< http://www.pucrs.br/mj/datas-comemorativas/transito/artigo-problemas-e-desafios-do-transporte-publicourbano.php >. Acesso em: 21 de agosto de 2013.

Metrô Rio (2013). Disponível em:< http://www.metrorio.com.br/index.htm>. Acesso em: 12 de dezembro de 2013.

Novaes, A. G. (1986). Sistemas de Transportes, vol 1: Análise da Demanda de Transporte. Editora Edgard Blucher Ltda.

Oliveira, J. G., Borges, F. H. e Jabbour, C. C., 2005, Avaliação de desempenho no âmbito da gestão ambiental na organização. In: XII Simpósio de Engenharia de Produção - SIMPEP, 2005, Bauru. XII Simpósio de Engenharia de Produção.

Ortuzar, J. d., \& Willumsen, L. G. (2011). Modelling Transport - Fourth edition. New York: Wiley\&Sons.

Pereira, C. M. C. (2007) Contribuição para a modelagem da divisão modal multinomial com base em estimativa de valor do tempo em transportes associada a um sistema de informação geográfica. Tese de doutorado. Universidade Federal do Rio de Janeiro - COPPE.

PDTU (2013) Atualização do Plano Diretor de Transporte Urbano da Região Metropolitana do Rio de Janeiro. Disponível em:< http://www.rj.gov.br/web/setrans/exibeconteudo?article-id=626280>. Acesso em: 14 de dezembro de 2013.

Pereira Neto, W. A. (2001) Modelo Multicritério de Avaliação de Desempenho Operacional do Transporte Coletivo por Ônibus no Município de Fortaleza. Fortaleza, 2001. XX, 192 fl., Dissertação de mestrado - Programa de Mestrado em Engenharia de Transportes, Centro de Tecnologia, Universidade Federal do Ceará, Fortaleza.

Pratt, R. H. \& Lomax, T. J. (1996). Performance measures for multimodal transportation systems. Transportation Research Record, Washington, v. 1518, p. 85-93.

Romero, B. de C. (2006). Análise da localização de plataformas logísticas: aplicação ao caso do ETSP Entreposto Terminal São Paulo - da CEAGESP. Dissertação de mestrado, USP, São Paulo

Roy, B. (1968). Clasement et choix en presence de points de vue multiples (La method Electre). RIRO 8, p. 5775 .

Roy, B. \& Bertier, P. (1973). La method Electre II: une application au mediaplanning. In: Ross, M. (Ed.) OR'72, North-Holland Publishing Company, p. $291-302$.

Roy, B. \& Hogonnard, J. C. (1982). Ranking of suburban line extension projects on the Paris metro system by multicriteria method. Transportation Research, v. 164A, n. 4, p. 301-312.

Roy, B. \& Skalka, J. M. (1985). Electre IS: aspects méthodologiques et guide d'utilisation. Cahier du LAMSADE, Paris: Université de Paris-Dauphine, n. 30.

Roy, B. (1978). Electre III: un algorithme de classements fondé sur une représenation floue des préferences em présence de critères multiples. Cah. Centre Etudes Rech. Opér., v. 20, pp. 3-24.

Saaty, T. L.(1980). The Analytic Hierarchy Process: Planning, Priority Setting, Resource Allocation. ISBN 0-07054371-2, McGraw-Hill.

Silva, E. L. \& Menezes, E. M. (2001). Metodologia da pesquisa e elaboração de dissertação. 3. ed. rev. atual.Florianópolis: Laboratório de Ensino a Distância da UFSC, 121 p.

Silva Neves, J. C (2000). Aplicação da Análise Envoltória de Dados (DEA) Para Avaliação de Fornecedores. Tese de mestrado em Sistemas e Computação - Instituto Militar de Engenharia.

Taha, H. A. (2008). Pesquisa Operacional: uma visão geral; tradução Arlete Simile Marques; revisão técnica Rodrigo Arnaldo Scarpel - 8a edição - São Paulo: Pearson Prentice Hall.

TARIFA DE TÁXI (2013). Disponível em:< http://www.tarifadetaxi.com/rio-de-janeiro >. Acesso em: 12 de dezembro de 2013.

Yu, W. (1992). Aide multicritère à la decision dans le cadre de la problématique du TRI. Tese de doutorado Université de Paris-Dauphine, Paris. 